\title{
Relation entre les propriétés chimique, cristallographique et électronique de films de sulfure de cadmium préparés par pulvérisation réactive
}

\author{
F. Dutault et J. Lahaye \\ Centre de Recherches sur la Physico-Chimie des Surfaces Solides, C.N.R.S. \\ 24, avenue du Président-Kennedy, 68200 Mulhouse, France.
}

(Reçu le 2 août 1979, révisé le 7 décembre 1979, accepté le 12 décembre 1979)

\begin{abstract}
Résumé. - Des films de sulfure de cadmium ont été préparés par pulvérisation réactive de chlorure de cadmium et de thiourée. Les auteurs décrivent l'influence du recuit sur la teneur en chlore et l'organisation cristalline du matériau en fonction de sa température de préparation. Ils établissent une corrélation entre ces deux transformations et la résistivité du film dont l'évolution est étudiée en fonction des températures de préparation et de recuit.

Abstract. - Films of cadmium sulfide were prepared by spray of aqueous solution of cadmium chloride and thiourea. Influences of preparation and post-treatment temperatures on chloride contents, crystalline organization and resistivity of films are described.
\end{abstract}

1. Introduction. - La pulvérisation réactive est une méthode économique d'élaboration de certains matériaux et en particulier de sulfure de cadmium destiné à la transformation photovoltaïque de la lumière. Cette méthode consiste à projeter sur un substrat chauffé un brouillard d'une solution de réactifs contenant les éléments nécessaires à la fabrication du matériau. Sous l'effet de la température, il y a réaction et formation sur le substrat d'un film du matériau désiré, en l'occurrence du sulfure de cadmium.

Ce procédé, promu par Chamberlin $\mathbf{R}$. R. et Skarman J. S. [1], est particulièrement adapté à l'élaboration en continu de grandes surfaces. Toutefois, le sulfure de cadmium ainsi obtenu possède des caractéristiques moins satisfaisantes que celui qui est préparé par évaporation sous pression réduite. Il convient donc d'améliorer les propriétés du matériau élaboré par pulvérisation. Or, à notre connaissance, les recherches effectuées ont consisté, le plus souvent, à faire varier empiriquement les paramètres expérimentaux. Nous avons préféré entreprendre une approche chronologique des phénomènes chimiques et physico-chimiques qui transforment la gouttelette de solution réactive en sulfure de cadmium. Celle-ci a comporté l'étude successive de l'aérosol, des composés intermédiaires formés et de leur décomposition ther- mique ainsi que du recuit du film. Ces différentes études constituent les travaux de thèse de l'un d'entre nous [2]. Dans le cadre du présent article, nous décrivons essentiellement l'effet du post-traitement thermique sur diverses caractéristiques des couches.

2. Appareillage. - 2.1 Elaboration DES FILMS. Les gouttelettes sont produites par un générateur d'aérosols monodisperses mis au point par Berglund R. N. et Liu B. Y. H. [3] et commercialisé par la Société T.S.I. (Thermo-Systems Inc.). Les caractéristiques expérimentales sont, dans le cas présent, les suivantes :

- Solution réactive : solution aqueuse équimolaire et décinormale de thiourée et de chlorure de cadmium.

- Générateur : gouttelettes de $140 \mu \mathrm{m}$ de diamètre, véhiculées par de l'azote sec.

- Température : la détection est assurée d'une manière relative par un thermocouple soudé sur un substrat témoin. Les écarts à l'équilibre, provoqués par le dépôt des gouttelettes, sont minimisés par un asservissement du système de chauffage.

- Film : le matériau est élaboré à une vitesse de $0,10 \pm 0,05 \mu \mathrm{m} / \mathrm{min}$., jusqu'à obtention d'un film de $2 \pm 1 \mu \mathrm{m}$ d'épaisseur moyenne, puis retiré du support chauffant dès la pulvérisation terminée. 
Les recuits sont effectués sous courant d'air préchauffé dans un four tubulaire. La température du four et la durée du traitement de la couche sont précisées à chaque manipulation.

2.2 CARACTÉRISATION DES FILMS. - Afin de comparer les caractéristiques chimiques, cristallographiques, morphologiques et électriques des matériaux élaborés, nous avons mis en œuvre des méthodes non destructives et les avons appliquées à la même couche.

La propriété chimique retenue pour caractériser le matériau est la teneur en chlore déterminée par fluorescence $X$; elle est exprimée en unités arbitraires par le rapport de l'intensité du pic du chlore (raie $\mathrm{K}_{\alpha}$ correspondant à un angle $\theta$ de $65,48^{\circ}$ pour le cristal choisi) à celle du pic du cadmium (raie $K_{\alpha}$ à $\theta=7,02^{\circ}$ ). L'appareil PHILIPS PW 1410 utilisé a les caractéristiques suivantes :

- anticathode de molybdène,

$-i=30 \mathrm{~mA}, V=50 \mathrm{kV}$,

- cristal analyseur de pentaérythrite,

- compteur proportionnel à flux gazeux (mélange argon-méthane).

En complément de cette technique qui ne permet pas de détecter les éléments légers, nous avons utilisé, dans un nombre limité de cas, l'analyse élémentaire pour mettre en évidence le carbone et l'azote.

Les caractéristiques cristallographiques ont été déterminées à partir de diffractogrammes de rayons $\mathbf{X}$ obtenus à l'aide d'un appareil PHILIPS 1010 $(i=20 \mathrm{~mA}, V=40 \mathrm{kV})$ muni d'un filtre de nickel qui sélectionne la raie $\mathrm{K}_{\alpha}$ du cuivre.

La mesure de l'intensité relative et de la largeur des raies nous renseigne respectivement sur l'orientation et la taille des cristallites. En effet, la formule de Scherrer permet de calculer la hauteur d'empilement des plans correspondant à la raie choisie [4] :

$$
l_{[h k l]}=\frac{K \lambda}{B_{l} \cos \theta}
$$

où $\lambda$ est la longueur d'onde du rayonnement incident, $\theta$ l'angle de Bragg, $B_{l}$ la contribution de la taille à l'élargissement de la raie à mi-hauteur, $K$ une constante choisie égale à 0,9 et $l$ la dimension moyenne des cristallites suivant la direction de la rangée $[h k l]$. Notons que d'autres facteurs provoquent l'élargissement des raies. Ce sont à la fois des facteurs instrumentaux et des facteurs structuraux. Nous avons soustrait la contribution instrumentale en calibrant l'appareil avec du chlorure de sodium dont les cristaux sont beaucoup plus gros que ceux qui nous intéressent. Par contre, nous n'avons pas tenu compte des imperfections cristallines qui contribuent certainement aussi à l'élargissement des raies.

Les propriétés électriques sont caractérisées par des mesures de résistivité effectuées, à l'obscurité et à température ambiante, à l'aide d'un mégohmmètre AUTOMATION INTERNATIONAL type W.E. 678 ou d'un appareil à quatre pointes JANDEL, alimenté par une source de courant KEITHLEY 227.

Enfin, ces déterminations ont été complétées par des observations en microscopie électronique à balayage effectuées avec un appareil JEOL.

3. Résultats expérimentaux. - Le solvant - l'eau dans notre cas - s'évapore sous l'effet conjugué de la température élevée et du faible degré hygrométrique du gaz vecteur choisi. Cette évaporation provoque la cristallisation d'un composé intermédiaire qui a été caractérisé comme étant le complexe de formule $\mathrm{CdCl}_{2}\left[\mathrm{SCN}_{2} \mathrm{H}_{4}\right]$ [5]. La morphologie du résidu de cristallisation, laquelle régit la morphologie du matériau final, peut être très différente d'un film à l'autre, comme certains auteurs l'ont déjà signalé [6] et comme nous l'avons expliqué par ailleurs [2] en précisant, à la fois par le calcul et l'expérience, les paramètres influençant la formation de sphères creuses ou de films homogènes.

Lorsque l'eau est éliminée, la température augmente brusquement et provoque la décomposition du complexe en sulfure de cadmium [7]. Toutefois, il reste, notamment à basse température de préparation, des hétéroatomes dans les couches. En effet, mis à part la présence de chlore relevée par plusieurs auteurs $[8,9]$, nous avons aussi détecté du carbone et de l'azote. Or, ces hétéroatomes disparaissent au cours du recuit. Cette disparition est progressive et dépend essentiellement de la température atteinte.

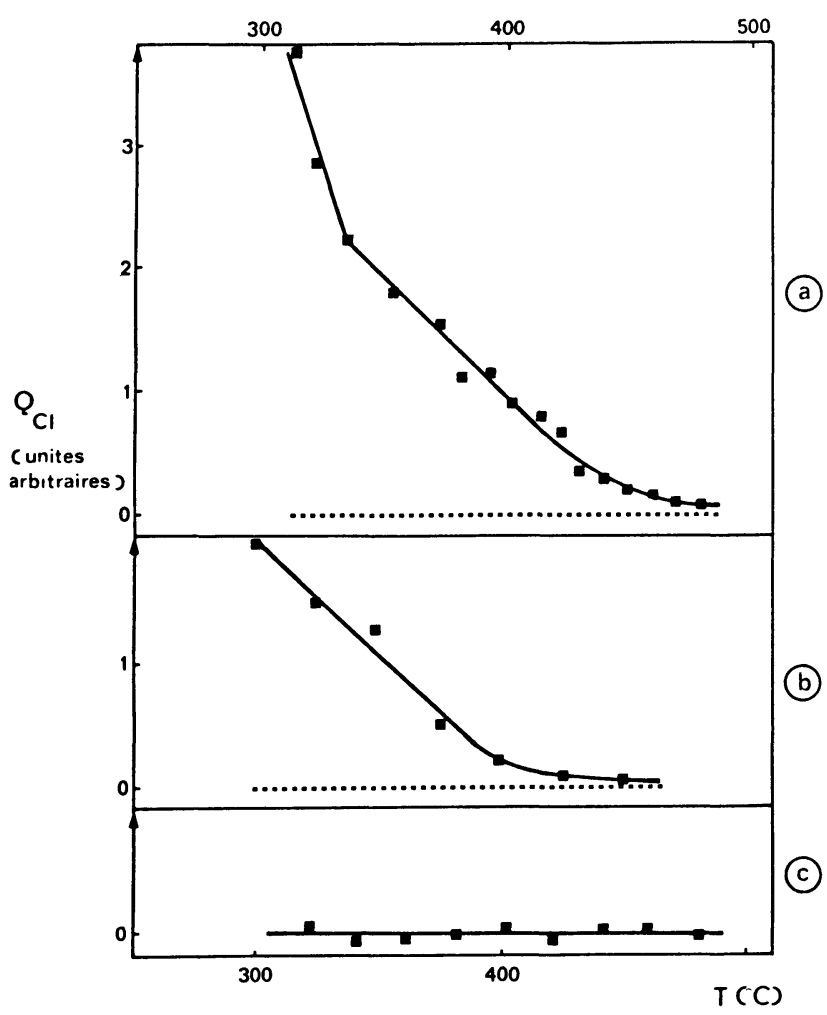

Fig. 1. - Quantité de chlore présente dans les films, en fonction des températures :

- de préparation : (a) en fin de pulvérisation: (c) après recuit d'une heure à $500^{\circ} \mathrm{C}$;

- de post-traitement : (b) recuits successifs d'une heure pour un film préparé à $260^{\circ} \mathrm{C}$. 
Elle s'accompagne de modifications importantes de la taille, de l'orientation des cristallites, de la morphologie et de la résistivité des films, comme nous allons le montrer dans le cadre de cet article.

3.1 COMPOSITION DES COUCHES. - Le rapport chlore/cadmium dans la couche (Fig. 1) est une fonction décroissante des températures de préparation (courbe 1a) et de traitement (recuit d'une heure suffisamment long pour être très proche de l'état d'équilibre) (courbe 1b). Il tend vers une valeur nulle (courbe 1c), à l'incertitude des résultats près, après un recuit à $500^{\circ} \mathrm{C}$.

Signalons que l'incertitude de toutes les mesures est assimilée à l'écart-type d'un échantillon de dix mesures effectuées sur une même couche, préparée à une température s'écartant de la moyenne, de $5{ }^{\circ} \mathrm{C}$ au maximum. Notons, d'autre part, que le décalage, observé entre les courbes obtenues en fonction des températures de préparation et des températures de recuit, est attribuable au caractère relatif de la détermination de la température de surface du substrat.

Comme nous l'avons déjà signalé, le chlore n'est pas la seule impureté présente dans les couches puisque nous avons aussi détecté du carbone et de l'azote. La variation de la teneur en ces deux éléments avec la température suit d'ailleurs celle du chlore (tableau I).

Tableau I. - Teneurs élémentaires exprimées en grammes pour 100 grammes de produit brut et déterminées en fonction de la température de préparation $T_{\mathrm{s}}$ et de recuit $T_{\mathrm{r}}$.

$\begin{array}{ccccc}T_{\mathrm{s}}\left({ }^{\circ} \mathrm{C}\right) & T_{\mathrm{r}}\left({ }^{\circ} \mathrm{C}\right) & \mathrm{Cl} & \mathrm{N} & \mathrm{C} \\ \frac{-}{320} & - & \frac{-}{5,2} & -0,8 & -0,6 \\ 320 & 380 & 4,3 & 0,5 & 0,5 \\ 380 & & 2,9 & 0,2 & 0,3 \\ 380 & 440 & & 0,1 & 0,1\end{array}$

3.2 Proprítés CRISTALlographiQues. - Les modifications chimiques précédemment citées sont accompagnées de transformations cristallographiques, tant sur le plan de la taille que sur celui de l'orientation des cristallites.

3.2.1 Taille des cristallites. - La taille des cristallites, qui passe de 20 à $60 \mathrm{~nm}$ lorsque la température de préparation passe de 300 à $500^{\circ} \mathrm{C}$, est modifiée de façon importante - du moins pour les températures les plus faibles - par un recuit d'une heure à $500^{\circ} \mathrm{C}$ (Fig. $2 b$ ). L'écart, sur la figure, entre la courbe en traits pleins et la droite en pointillés, traduit l'augmentation de taille des cristallites. Cette augmentation de taille semble quantitativement liée à la diminution du taux de chlore représentée sur la figure $2 a$.

3.2.2 Orientation des cristallites. - Parallèlement, l'orientation préférentielle des cristallites sur le substrat subit, par le même recuit, une modification en corrélation avec les précédentes (Fig. $2 c$ ). Notons

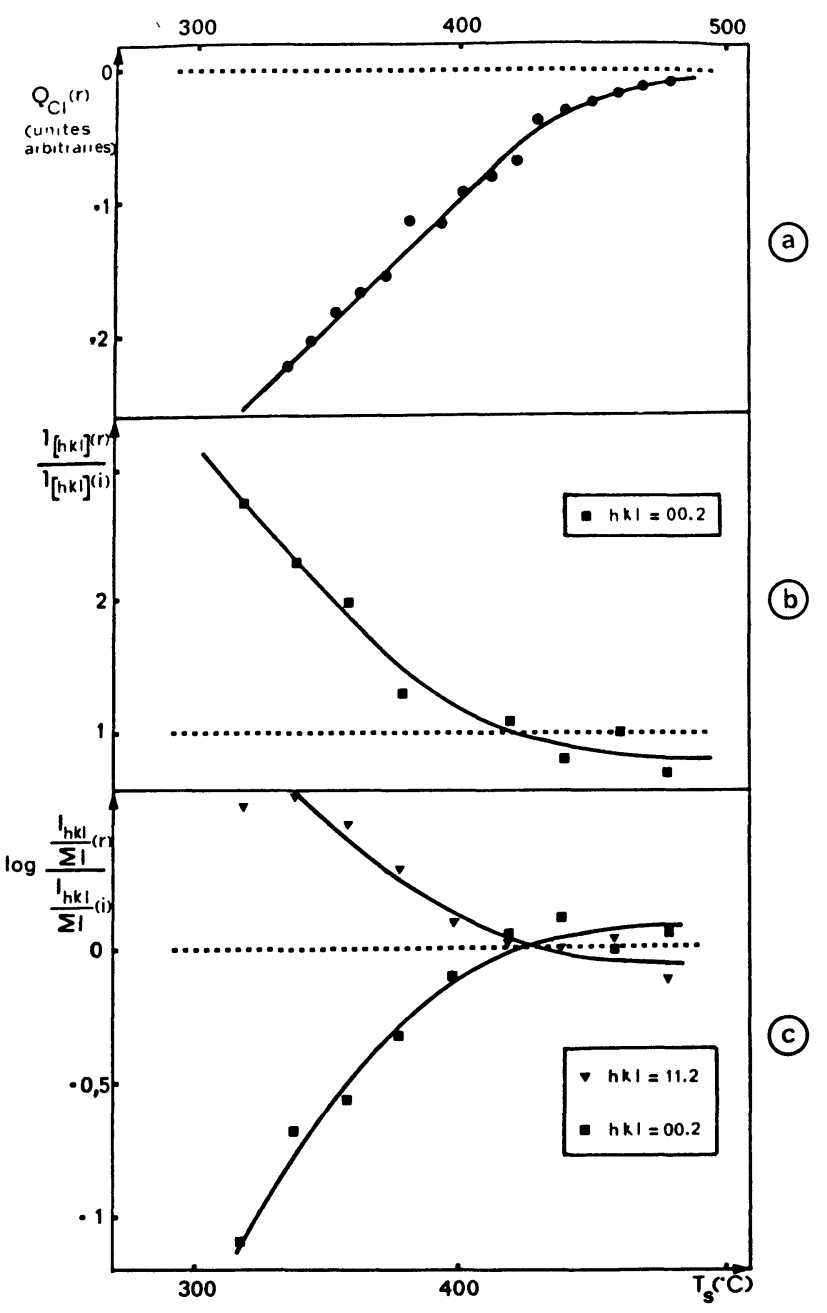

Fig. 2. - Corrélation entre le départ de chlore (a), l'augmentation de taille des cristallites (b) et leur orientation sur le substrat (c), en fonction de la température de préparation $T_{\mathrm{s}}$, avant (courbes en pointillés) et après recuit (courbes en traits pleins). a) $Q_{\mathrm{n}}$ : quantité de chlore: b) ' ${ }_{[h k l]}:$ longueur des cristallites suivant la direction de la rangee $[h k l]$; c) $I_{[h k l]} / \Sigma I:$ intensité relative d'un pic du diffractogramme de rayons $\mathrm{X} ; r$ : après recuit, $i$ : dès la pulvérisation terminée.

que l'orientation préférentielle généralement recherchée - rangée [00.2] perpendiculaire au substrat disparaît au profit de celle où les plans (11.2) sont parallèles à ce même substrat.

3.2.3 Morphologie des couches. - Les couches subissent également par recuit des modifications observables en microscopie électronique à balayage (clichés 1 à 4). En effet, on distingue, en surface du film, des particules pouvant avoir des tailles supérieures au micron et qui présentent, dans certains cas, des formes géométriques bien définies (domaines cristallins).

3.3 RÉSISTIVITÉ DES COUCHES. - Des travaux entrepris par Savelli M. et ses collaborateurs [10] ont consisté à étudier la variation de la résistivité d'une couche en fonction de la température de recuit. La méthode est intéressante et nous avons essayé de la 
transposer à notre étude. Pour cela, nous avons recuit par paliers successifs de dix degrés, des couches préparées à différentes températures. Entre chaque palier de traitement qui dure dix minutes, la couche est retirée du four et la résistivité mesurée. Chacune de ces mesures - moyenne de plusieurs déterminations en différents points de la couche - est reportée sur la figure 3. Pour ne pas surcharger cette figure, nous n'avons pas mentionné les incertitudes sur les valeurs moyennes de la résistivité et sur la température respectivement inférieures au dixième de la mantisse et à $5^{\circ} \mathrm{C}$. Signalons, d'autre part, que l'allure des courbes est reproductible dans le cas de couches élaborées dans les mêmes conditions - la valeur de la résistivité pouvant toutefois varier d'une puissance de 10 entre deux couches identiques.

Cette série de courbes nous montre que la résistivité dépend à la fois de la température de préparation et de la température de recuit. De plus, il est intéressant de noter que ces courbes présentent, du moins dans le cas de faibles températures de préparation, une analogie avec la courbe établie en fonction de la température de préparation par $\mathrm{Ma} \mathrm{Y}$. Y. et Bube R. H. [11]. Par contre, l'allure des courbes établies lors d'une seconde série de recuits dans les mêmes conditions est très différente (Fig. 4). Nous donnons une interprétation de la forme de ces courbes dans la dernière partie de l'article.

4. Interprétation et discussion des résultats. -4.1 INTERPRÉTATION. - La présence de l'élément chlore dans les couches pourrait être attribuée à la cristallisation de chlorure de cadmium en cours de pulvérisation. Cette interprétation serait en accord avec la composition de la solution réactive et avec nos précédents résultats qui montrent que la cristallisation de ce sel est d'autant plus importante que la température de cristallisation [5] ou de décomposition [7] est plus faible. De plus, le chlore, tout comme une partie du cadmium, est soluble dans l'eau dans un rapport stœchiométrique qui correspond à celui du chlorure de cadmium [8].

Toutefois, cette hypothèse doit être abandonnée car le chlore est en grande partie libéré lors du recuit. Le chlorure de cadmium est thermiquement stable dans le domaine de températures utilisées et, par conséquent, il ne peut s'agir de ce composé. La disparition simultanée de carbone et d'azote est conforme à l'existence d'une solution solide entre le sulfure de cadmium et le complexe non encore dissocié. Cette hypothèse permettrait d'expliquer la corrélation entre la teneur en hétéroéléments et la température maximale de traitement de la couche.

Une autre caractéristique intéressante est la forme de la courbe donnant la résistivité en fonction de la température de post-traitement. Nous allons chercher à interpréter la série de minimums et de maximums de la figure 3.

Lorsque le post-traitement thermique est effectué
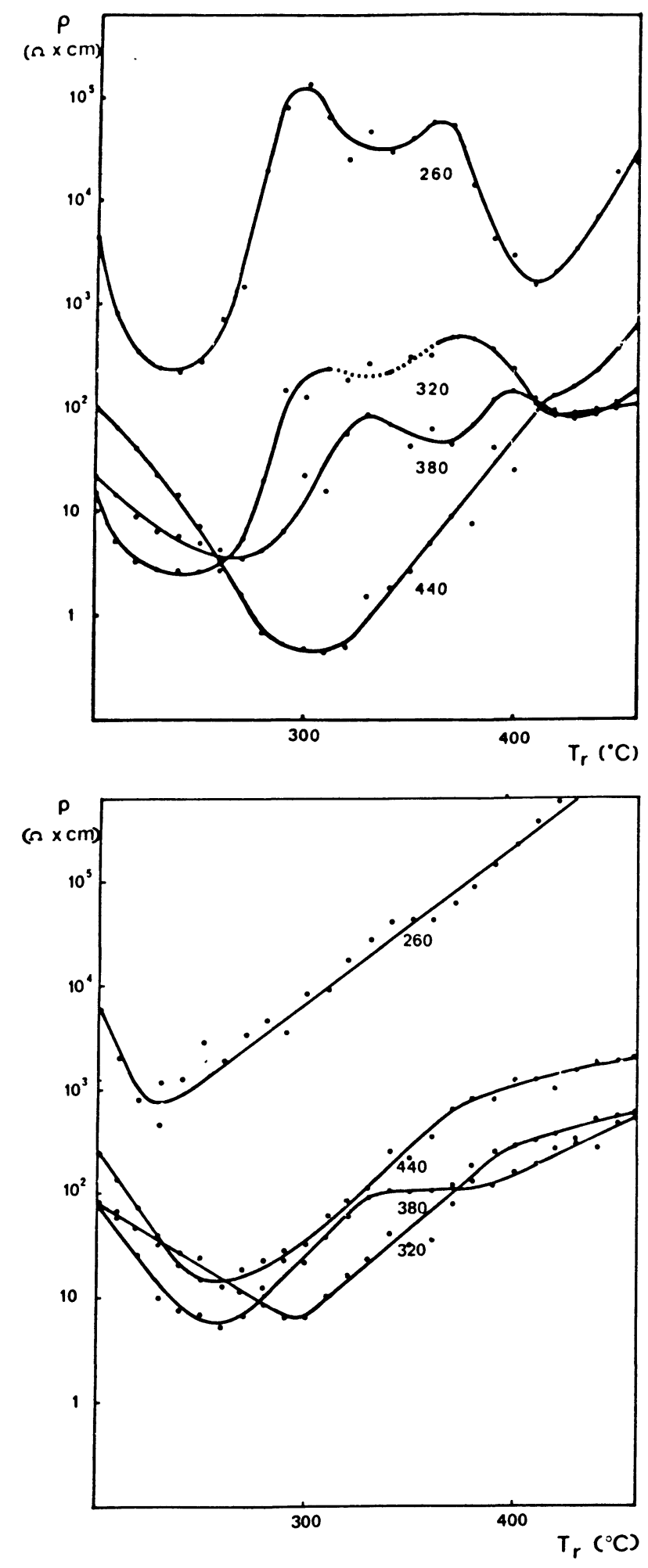

Figs. 3 et 4 . - Evolution de la résistivité de films préparés à 260 , 320,380 et $440^{\circ} \mathrm{C}$ lors de recuits successifs à température $T_{\mathrm{r}}$ croissante.

Fig. 3. - Première série de recuits.

Fig. 4. - Deuxième série de recuits.

sous pression réduite [12] ou sous atmosphère d'azote [10] ou d'hydrogène [9], la première chute de résistivité est attribuée à la désorption d'oxygène. En effet, l'oxygène chimisorbé diminue à la fois le nombre de 

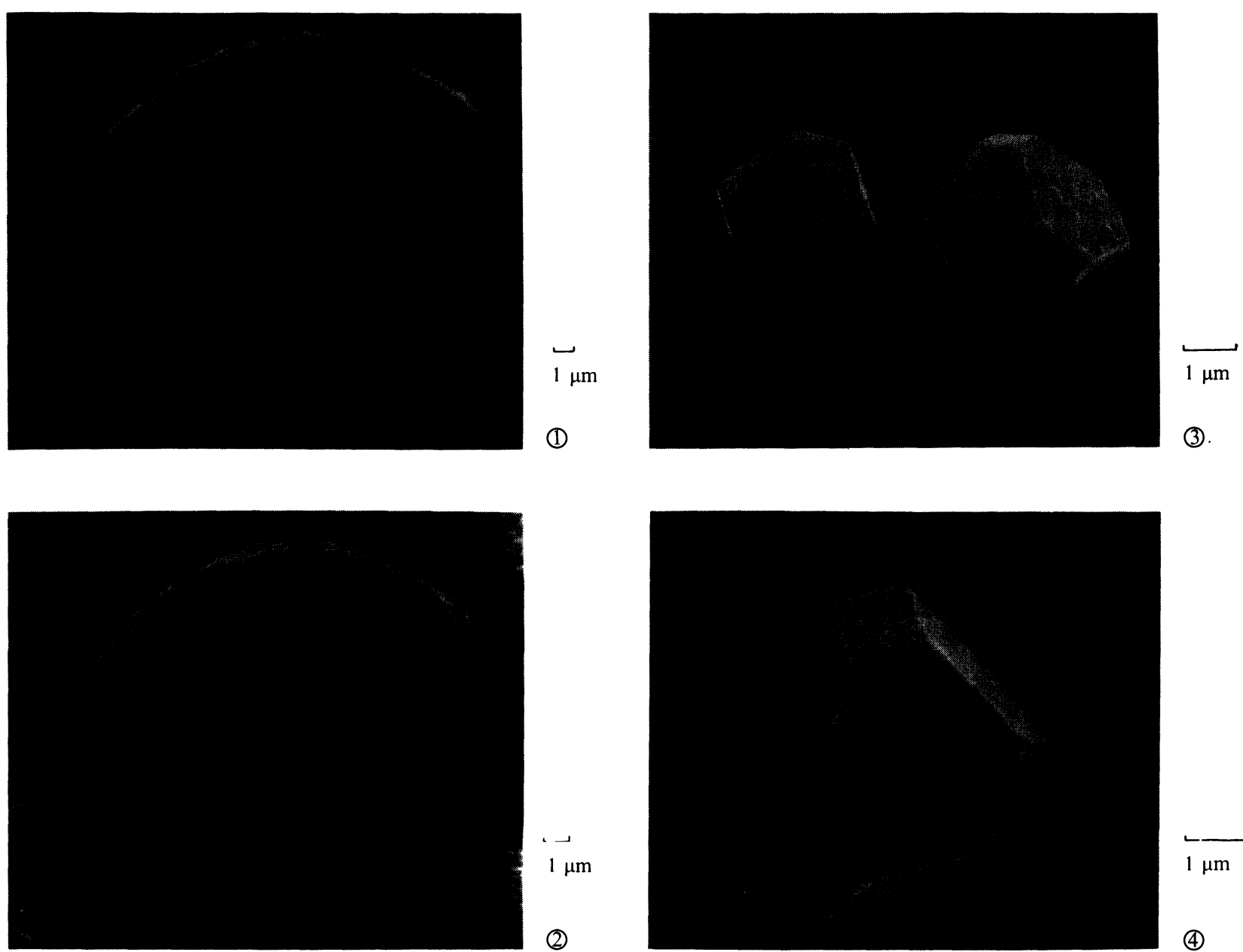

Clichés 1 et 2. - Observations par microscopie électronique à balayage de la surface d'un film sans post-traitement (1) et après recuit (2). Température de préparation : $400^{\circ} \mathrm{C}$. Diamètre des gouttelettes : $70 \mu \mathrm{m}$. Vitesse d'élaboration : $0,4 \mu \mathrm{m} / \mathrm{h}$. Epaisseur moyenne : $2,1 \mu \mathrm{m}$.

porteurs (en agissant comme impureté acceptrice) et la mobilité de ces porteurs (en augmentant la hauteur de la barrière de potentiel entre les grains). La décroissance, qui se poursuit généralement jusqu'à une température comprise entre 240 et $300^{\circ} \mathrm{C}$, peut être expliquée par le même phénomène. Toutefois, il faut s'attendre à une diminution moins importante dans ce cas, où la pression partielle d'oxygène n'est pas nulle.

Nous ne nous sommes pas attardés à l'origine du second minimum qui n'est d'ailleurs pas toujours très net. Il pourrait s'agir, vu la température à laquelle il intervient, de la sublimation de chlorure d'ammonium.

Par contre, nous nous sommes plus spécialement intéressés à la troisième décroissance présentant un minimum à une température comprise entre 410 et $430^{\circ} \mathrm{C}$. Cette diminution de la résistivité est d'autant plus précoce et d'autant plus importante que la température de préparation est plus faible. Elle est causée par un phénomène irréversible - contrairement à la

Clichés 3 et 4 . - Observations par microscopie électronique à balayage de la surface d'un film préparé très lentement, et en conséquence, ayant subi un auto-recuit. Température de préparation : $400^{\circ} \mathrm{C}$. Diamètre des gouttelettes : $23 \mu \mathrm{m}$. Vitesse d'élaboration : $0,07 \mu \mathrm{m} / \mathrm{h}$. Epaisseur moyenne : $1 \mu \mathrm{m}$.

désorption de l'oxygène - comme nous pouvons le constater sur les courbes de la figure 4 établies lors d'une seconde série de recuits effectuée dans les mêmes conditions. Il semble donc que la disparition du troisième minimum puisse résulter de l'appauvrissement en hétéroéléments et de l'augmentation de la taille des cristallites.

Jusqu'à présent, nous nous sommes essentiellement intéressés aux chutes de résistivité provoquées par l'augmentation de la température de traitement. L'interprétation de ces accidents ne suffit pas pour expliquer l'allure générale des courbes et en particulier l'existence de minimums. Nous serions tentés de l'attribuer à une augmentation continue de la résistivité, provọquée par un phénomène tel qu'un deuxième type de fixation de l'oxygène [13] conduisant à la formation d'oxyde de cadmium.

4.2 Discussion. - Nous venons de montrer que l'accroissement de température pouvait être responsable de nombreuses transformations dans les couches. 
Il est intéressant d'essayer de corréler les phénomènes observés.

Le départ de chlore provient de l'instabilité thermique d'espèces intermédiaires et peut être considéré pour cette raison comme indépendant des autres phénomènes. Il convient d'être beaucoup plus prudent dans l'interprétation des modifications cristallographiques. Celles-ci sont d'autant plus importantes que la quantité de chlore initialement présente est plus grande. Toutefois, elles ne conduisent pas à des orientations comparables à celles obtenues dans le cas de couches préparées directement à haute température. En conséquence, on peut considérer que le changement de cristallinité résulte, au moins en partie, du départ de l'élément chlore, bien que l'effet propre de la température sur la recristallisation ne puisse être rejeté. Quant à la résistivité, elle est certainement influencée, comme nous l'avons déjà signalé, par les deux modifications précédentes.

L'ensemble de cette discussion est résumé dans le schéma suivant qui précise les corrélations entre les différents phénomènes et le rôle prépondérant du chlore.

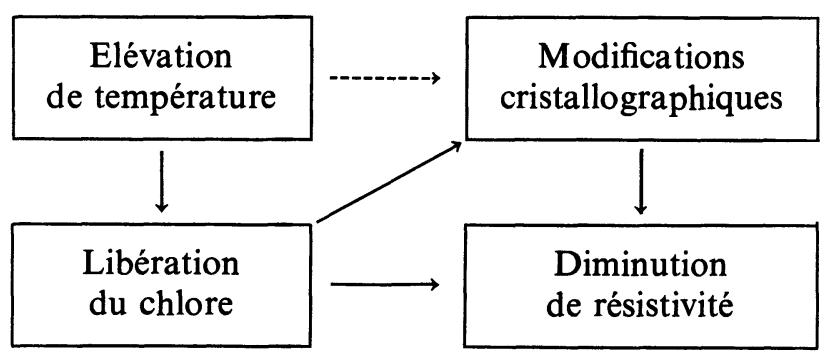

5. Conclusion. - Le recuit est capable de modifier de nombreuses caractéristiques de films de sulfure de cadmium préparés, particulièrement à basse température, par pulvérisation réactive. Le choix des températures de préparation et de post-traitement permet de moduler les transformations et d'élargir ainsi l'éventail de l'échantillonnage de films réalisables.

\section{Bibliographie}

[1] Chamberlin, R. R. et Skarman, J. S., J. Electrochem. Soc 1 (1966) 86-9.

[2] Dutault, F., Thèse de Docteur-Ingénieur, Mulhouse (1979).

[3] Berglund, R. N. et Liu, B. Y. H., Environ. Sci. Technol. 7 (1973) 147-53.

[4] Anderson, J. R., Structure of Metallic Catalysts (Academic Press, London, New York, San Francisco) 1975, p. 364-9.

[5] Dutault, F. et Lahaye, J., Bull. Soc. Chim. France 1 5-6 (1979) 145-9.

[6] Berg, R. S., NaSby, R. D. et Lampkin, C., J. Vac. Sci. Technol. 15 (1978) 359-62.

[7] Dutault, F. et Lahaye, J., Publication à paraître.

[8] Kerm, K. B., Tr. Tallin. Politekh. Inst., Ser. A 323 (1972) $39-43$.
[9] Bougnot, J., Perotin, M., Marucchi, J. et Savelli, M., Convention C.C.E., Bruxelles, $n^{0}$ 430-78-1 ES.F, Rapport $\mathrm{n}^{\circ} 2,30$ juin 1978 .

[10] Bougnot, J., Perotin, M., Marucchi, J. et Savelli, M., Convention C.E.E., Bruxelles, $n^{0}$ 159-76-7 ES.F, Rapport final d'activité, 31 décembre 1977.

[11] MA, Y. Y. et Bube, R. H., J. Electrochem. Soc. 124 (1977) $1430-5$.

[12] Martinuzzi, S., Cabane-Brouty, F., Oualid, T., Gervais, J., Mostavan, A. et Granier, J. L., Proc. of Int. Conf., Luxembourg, 27-30 septembre 1977.

[13] Benton, A. F. et White, T. A., J. Am. Chem. Soc. 52 (1930) 2325. 